Draft Version OCtober 2, 2018

Preprint typeset using $\mathrm{LAT}_{\mathrm{E} X} \mathrm{X}$ style emulateapj v. 12/16/11

\title{
THE PROMPT EMISSION OF GAMMA-RAY BURSTS FROM THE WIND OF NEWBORN MILLISECOND MAGNETARS: A CASE STUDY OF GRB 160804A
}

\author{
Di XiaO ${ }^{1,2}$, Zong-KAi Peng ${ }^{1,2}$, Bin-Bin Zhang ${ }^{1,2}$, And Zi-GaO DAi ${ }^{1,2}$ \\ ${ }^{1}$ School of Astronomy and Space Science, Nanjing University, Nanjing 210093, China; dxiao@nju.edu.cn; bbzhang@nju.edu.cn; \\ dzg@nju.edu.cn and \\ ${ }^{2}$ Key Laboratory of Modern Astronomy and Astrophysics (Nanjing University), Ministry of Education, China \\ Draft version October 2, 2018
}

\begin{abstract}
In this paper, we revisit the scenario that an internal gradual magnetic dissipation takes place within the wind from a newborn millisecond magnetar can be responsible for gamma-ray burst production. We show that a combination of two emission components in this model, i.e., the photospheric emission from the wind and the synchrotron radiation within the magnetic reconnection region, can give a reasonable fit to the observed spectrum of the prompt emission phase of GRB 160804A. We obtain the physical parameters through a Monte Carlo procedure and deduce the initial spin period and magnetic field of the central magnetar. Furthermore, the independent afterglow fitting analysis gives a consistent result, adding great credibility to this scenario. In addition, we predict a subclass of GRBs called bursts from such a Magnetar wind Internal Gradual MAgnetic Dissipation (abbreviated as "MIGMAD bursts") that have several distinctive properties.
\end{abstract}

Subject headings: gamma-ray bursts: general - radiation mechanisms: non-thermal

\section{INTRODUCTION}

Millisecond magnetars are widely believed to be one of the possible central engines of gamma-ray bursts (for recent reviews, see Faber \& Rasio 2012; Kumar \& Zhang 2015; Dai et al. 2017), either from the collapse of massive stars or double neutron star mergers. After formation, a proto-magnetar ejects an outflow and produces a gamma-ray burst (GRB). Generally, for a magnetar central engine, there are two kinds of outflow. One is driven due to accretion of the magnetar and the other is the proto-magnetar wind. The former extracts the gravitational energy of accreted matter and can reach a high luminosity to power a normal GRB that is similar to a black hole central engine(e.g. Zhang \& Dai 2008, 2009, 2010) while the latter extracts the stellar rotational energy and is usually less luminous (Usov 1992; Dai \& Lu 1998a,b). There are at least two indirect observational evidences for the existence of such a wind. First, a pulsar wind nebula is suggested to originate from the shock produced as the pulsar wind interacts with an interstellar medium. For instance, the measured radio spectrum of the Crab Nebula is naturally explained if a wind with a Lorentz factor of $\sim 10^{4}$ from the Crab pulsar is introduced (Atoyan 1999). Second, a bunch of GRB X-ray afterglows exhibit plateau or flare features that can be well explained by the long-term energy injection from central engines (e.g. Zhang et al. 2006). The injected energy is possibly from the spin-down power that is mediated by the wind ${ }^{1}$ (e.g. Yu et al. 2009, 2010; Lü et al. 2018). This proto-magnetar wind is initially cold and Poynting-flux dominated (Metzger et al. 2011). Later, as the magnetic

1 Note that there are some instances that are not in favor of magnetar's energy injection (Beniamini \& Mochkovitch 2017). The other possible kind of injected energy comes from the fallback accretion of the black hole system (e.g. Ruffert et al. 1997; Rosswog et al. 2003; Lei et al. 2013; Wu et al. 2013). However, the fall-back mass may be too large explaining the plateau or flare at very late times ( $>10^{6} \mathrm{~s}$ ) (for a recent review, see Liu et al. 2017). energy being converted to the kinetic energy of bulk motion, this wind turns to be electron-positron dominated that can further energize the interstellar medium. In this process, very high-energy gamma-rays can be produced, as confirmed by the Fermi-LAT observation of Crab pulsar (Aharonian et al. 2012). Motivated by this, we would like to study the high-energy emission from the magnetic energy dissipation in the wind of the newborn magnetar.

The mechanism of GRB prompt emission has not been well known. In the standard model, it is attributed to the synchrotron emission from a bunch of accelerated electrons by internal shocks (Rees \& Mészáros 1994; Kobayashi et al. 1997; Daigne \& Mochkovitch 1998). Also, acceleration could be realized via abrupt magnetic reconnection (Zhang \& Yan 2011). However, the synchrotron model usually suffers from the low energy index problem, i.e., the low energy photon index $(\alpha)$ fitted by the Band function is often found close to -1 or even larger. Therefore we need additional physics to explain the observed spectrum. Possible approaches include involving marginally slow cooling regime of radiating electrons (Kumar \& McMahon 2008; Daigne et al. 2011; Beniamini \& Piran 2013; Beniamini et al. 2018), inverse Compton scattering (Bošnjak et al. 2009; Daigne et al. 2011; Geng et al. 2018) or decay of a magnetic field behind the shock (Derishev 2007; Lemoine 2013; Uhm \& Zhang 2014). Alternatively, the dissipative photospheric model can reproduce $\alpha \sim-1$ spectrum if dissipative mechanisms (e.g. internal shocks, magnetic reconnection, etc) occur just below the photosphere radius (Thompson 1994; Ghisellini \& Celotti 1999; Rees \& Mészáros 2005; Pe'er et al. 2005; Beloborodov 2010; Giannios 2012; Lundman et al. 2013; Deng \& Zhang 2014; Bégué \& Pe'er 2015; Gao \& Zhang 2015). Another scenario involves the internal gradual magnetic dissipation mechanism which suggests that the magnetic energy of a highly magnetized outflow can be converted 
to thermal energy and bulk kinetic energy via gradual dissipation (Spruit et al. 2001; Drenkhahn 2002; Drenkhahn \& Spruit 2002; Giannios \& Spruit 2005; Metzger et al. 2011; Giannios 2012; Beniamini et al. 2017). In the meantime, magnetic reconnection is able to accelerate electrons and produce non-thermal radiation (Beniamini \& Piran 2014). Observationally, one would expect superposition of thermal and non-thermal components that may lead to a hard low-energy spectral index (Beniamini \& Giannios 2017). Based on this consideration, in this paper we revisit the scenario that involves magnetic dissipation within the wind of a newborn magnetar as a possible mechanism for producing GRBs, which is named the Magnetar wind Internal Gradual MAgnetic Dissipation model (abbreviated as MIGMAD model and hereafter).

This paper is organized as follows. In Section 2 we introduce the MIGMAD model and predict its emission. Then, we perform a comprehensive case study of GRB 160804A and discuss some implications of its central magnetar in Section 3. At last, we present our conclusions in Section 4.

\section{THE MIGMAD MODEL}

A newborn millisecond magnetar is one of the possible central engines of both long and short GRBs. A wind from this proto-magnetar should be squeezed and collimated into a narrow jet while propagating in the envelope material, and finally penetrate out since the wind is long-lasting comparing to its break-out time (Bromberg et al. 2011, 2014; Bromberg \& Tchekhovskoy 2016). This wind is initially Poynting-flux dominated (Coroniti 1990) and its magnetic energy can be converted to thermal emission and bulk kinetic energy via internal gradual magnetic dissipation (Spruit et al. 2001; Drenkhahn 2002; Drenkhahn \& Spruit 2002; Giannios \& Spruit 2005). In the meantime, electrons can be accelerated by reconnection. Particle-in-Cell (PIC) simulations suggest that these accelerated electrons could have a power-law distribution with an index $p$ (Sironi \& Spitkovsky 2014; Guo et al. 2015; Kagan et al. 2015; Werner et al. 2016). Therefore, synchrotron emission is also expected from these electrons (Beniamini \& Piran 2014; Beniamini \& Giannios 2017). We calculate each component in this section.

At a given radius, the Poynting-flux luminosity of the wind could be written as (Giannios \& Spruit 2005; Beniamini \& Giannios 2017)

$$
L_{B}=c \frac{(r B)^{2}}{4 \pi}=L_{0}\left[1-\frac{\Gamma(r)}{\Gamma_{\text {sat }}}\right],
$$

where $L_{0}$ is the total luminosity of the wind (per steradian), and $B$ and $\Gamma(r)$ are the magnetic field strength and Lorentz factor of the wind at radius $r$ respectively. $\Gamma_{\text {sat }}$ is the bulk Lorentz factor of the wind at the saturation radius that is given by $r_{\text {sat }}=\lambda \Gamma_{\text {sat }}^{2} /(6 \epsilon)=$ $1.7 \times 10^{15} \Gamma_{\text {sat }, 4}^{2}(\lambda / \epsilon)_{8} \mathrm{~cm}$ (Beniamini \& Giannios 2017), where the typical "wavelength" of the field is $\lambda=$ $c P=3 \times 10^{7} P_{-3} \mathrm{~cm}$ in the striped wind configuration (Coroniti 1990; Spruit et al. 2001; Drenkhahn 2002; Drenkhahn \& Spruit 2002) and $\epsilon \sim 0.1-0.25$ is the ratio of the reconnection velocity to the speed of light (Guo et al. 2015; Liu et al. 2015). Since the co-moving temperature decreases as $T^{\prime} \propto r^{-7 / 9}$, the thermal luminosity decreases as $L_{\mathrm{th}}(r) \propto r^{-4 / 9}$ (Giannios \& Spruit $2005)$, substituting the energy dissipation rate $d \dot{E}=$ $-\left(d L_{B} / d r\right) d r$, then the total thermal photospheric luminosity can be obtained by integrating from the initially launching radius to the photospheric radius $r_{\mathrm{ph}}$ (Giannios \& Spruit 2005; Beniamini \& Giannios 2017; Xiao \& Dai 2017),

$$
\begin{aligned}
L_{\mathrm{ph}} & =\int_{0}^{r_{\mathrm{ph}}} \frac{1}{2}\left(\frac{r}{r_{\mathrm{ph}}}\right)^{4 / 9} d \dot{E} \\
& =2.6 \times 10^{47} L_{0,50}^{6 / 5} \Gamma_{\mathrm{sat}, 4}^{-1}\left(\frac{\lambda}{\epsilon}\right)_{8}^{-1 / 5} \operatorname{erg~s}^{-1} \mathrm{sr}^{-1},
\end{aligned}
$$

with the temperature being

$$
T_{\mathrm{ph}}=95 L_{0,50}^{1 / 10} \Gamma_{\mathrm{sat}, 4}^{1 / 4}\left(\frac{\lambda}{\epsilon}\right)_{8}^{-7 / 20} \mathrm{keV},
$$

where $r_{\mathrm{ph}}$ can be obtained by setting the Thomson scattering depth $\tau\left(r_{\mathrm{ph}}\right)=1$, which gives $r_{\mathrm{ph}}=3.0 \times$ $10^{9} L_{0,50}^{3 / 5} \Gamma_{\text {sat }, 4}^{-1}(\lambda / \epsilon)_{8}^{2 / 5} \mathrm{~cm}$ (Beniamini \& Giannios 2017; Xiao \& Dai 2017). So the thermal component can be written as

$$
L_{\nu}^{\mathrm{ph}}(\nu)=L_{\nu}^{\mathrm{ph}}\left(\nu ; L_{0}, \lambda / \epsilon, \Gamma_{\mathrm{sat}}\right) .
$$

In order to obtain the synchrotron spectrum, we need to calculate the relevant break frequencies. The three break frequencies $\nu_{m}, \nu_{c}, \nu_{a}$ depend on radius and can be obtained by the same way as in Xiao \& Dai (2017). $\nu_{\max }$ is the maximum frequency corresponds to the maximum electron Lorentz factor that is determined by equaling the synchrotron energy loss timescale with reconnection acceleration timescale. Initially the electrons are in the fast cooling regime for which the spectrum is (Sari et al. 1998)

$$
L_{\nu}^{\mathrm{syn}}= \begin{cases}L_{\nu, \max }^{\mathrm{syn}}\left(\nu / \nu_{c}\right)^{1 / 3} & \text { if } \nu<\nu_{c}, \\ L_{\nu, \max }^{\mathrm{syn}}\left(\nu / \nu_{c}\right)^{-1 / 2} & \text { if } \nu_{c}<\nu<\nu_{m}, \\ L_{\nu, \max }^{\mathrm{syn}}\left(\nu_{m} / \nu_{c}\right)^{-1 / 2}\left(\nu / \nu_{m}\right)^{-p / 2} & \text { if } \nu_{m}<\nu<\nu_{\max }\end{cases}
$$

where

$$
L_{\nu, \max }^{\mathrm{syn}}=\frac{m_{e} c^{2} \sigma_{T} \Gamma B^{\prime} N_{e}(r)}{3 q},
$$

with $N_{e}(r)$ being the total number of emitting electrons in the wind at $r$. Letting $\nu_{m}=\nu_{c}$, we can get the radius $r_{\text {tr }}$ at which the transition from fast cooling to slow cooling happens. Since usually $\nu_{a}>\nu_{c}$ holds at $r_{\mathrm{ph}}$, we can define another critical radius $r_{\mathrm{cr}}$ at which $\nu_{a}$ crosses $\nu_{c}$. Then the whole synchrotron spectrum can be written as follows (Xiao \& Dai 2017). Initially for $r_{\mathrm{ph}}<r \leq r_{\mathrm{cr}}$,

$$
L_{\nu}^{\text {syn }}= \begin{cases}L_{\nu_{a}}^{\operatorname{syn}}\left(\nu / \nu_{a}\right)^{11 / 8} & \text { if } \nu<\nu_{a}, \\ L_{\nu_{a}}^{\operatorname{syn}}\left(\nu / \nu_{a}\right)^{-1 / 2} & \text { if } \nu_{a}<\nu<\nu_{m}, \\ L_{\nu_{a}}^{\operatorname{syn}}\left(\nu_{m} / \nu_{a}\right)^{-1 / 2}\left(\nu / \nu_{m}\right)^{-p / 2} & \text { if } \nu_{m}<\nu<\nu_{\max } .\end{cases}
$$


Further for $r_{\mathrm{cr}} \leq r \leq r_{\mathrm{tr}}$,

$$
L_{\nu}^{\mathrm{syn}}= \begin{cases}L_{\nu_{a}}^{\mathrm{syn}}\left(\nu / \nu_{a}\right)^{11 / 8} & \text { if } \nu<\nu_{a}, \\ L_{\nu_{a}}^{\mathrm{syn}}\left(\nu / \nu_{a}\right)^{1 / 3} & \text { if } \nu_{a}<\nu<\nu_{c}, \\ L_{\nu_{a}}^{\mathrm{syn}}\left(\nu_{c} / \nu_{a}\right)^{1 / 3} & \text { if } \nu_{c}<\nu<\nu_{m}, \\ \times\left(\nu / \nu_{c}\right)^{-1 / 2} & \\ L_{\nu_{a}}^{\mathrm{syn}}\left(\nu_{c} / \nu_{a}\right)^{1 / 3} & \text { if } \nu_{m}<\nu<\nu_{\max } \\ \times\left(\nu_{m} / \nu_{c}\right)^{-1 / 2}\left(\nu / \nu_{m}\right)^{-p / 2}\end{cases}
$$

Lastly, for $r_{\mathrm{tr}} \leq r \leq r_{\mathrm{sat}}$,

$$
L_{\nu}^{\text {syn }}= \begin{cases}L_{\nu_{a}}^{\text {syn }}\left(\nu / \nu_{a}\right)^{11 / 8} & \text { if } \nu<\nu_{a}, \\ L_{\nu_{a}}^{\text {syn }}\left(\nu / \nu_{a}\right)^{1 / 3} & \text { if } \nu_{a}<\nu<\nu_{m}, \\ L_{\nu_{a}}^{\text {syn }}\left(\nu_{m} / \nu_{a}\right)^{1 / 3} & \text { if } \nu_{m}<\nu<\nu_{c}, \\ \times\left(\nu / \nu_{m}\right)^{-(p-1) / 2} & \\ L_{\nu_{a}}^{\text {syn }}\left(\nu_{m} / \nu_{a}\right)^{1 / 3} & \text { if } \nu_{c}<\nu<\nu_{\max } .\end{cases}
$$

The non-thermal synchrotron spectrum can be obtained by integrating the above expressions from the photospheric radius to the saturation radius. So the nonthermal component can be given by

$$
L_{\nu}^{\mathrm{syn}}(\nu)=L_{\nu}^{\mathrm{syn}}\left(\nu ; L_{0}, \lambda / \epsilon, \epsilon_{e}, \Gamma_{\mathrm{sat}}, p\right)
$$

where $\epsilon_{e}$ is the fraction of dissipated energy per electron.

Lastly, the total spectrum is the sum of the thermal and non-thermal fluxes,

$$
\begin{aligned}
F_{\nu}(\nu) & =F_{\nu}\left(\nu ; L_{0}, \lambda / \epsilon, \epsilon_{e}, \Gamma_{\mathrm{sat}}, p\right) \\
& =\frac{L_{\nu}^{\mathrm{ph}}\left(\nu ; L_{0}, \lambda / \epsilon, \Gamma_{\mathrm{sat}}\right)+L_{\nu}^{\mathrm{syn}}\left(\nu ; L_{0}, \lambda / \epsilon, \epsilon_{e}, \Gamma_{\mathrm{sat}}, p\right)}{4 \pi D_{L}^{2}}
\end{aligned}
$$

where $D_{L}$ is the luminosity distance.

\section{APPLICATION TO GRB 160804A}

GRB 160804A is a long gamma-ray burst that triggered both Fermi-GBM and Swift-BAT. It has a duration of $T_{90} \sim 130 \mathrm{~s}$ and peak energy $E_{p} \sim 100 \mathrm{keV}$ (Bissaldi et al. 2016; Marshall et al. 2016), which match the expectations of the MIGMAD model well. The afterglow emission of GRB 160804A is discovered by several groups and the absorption and emission line features in the optical afterglow suggests a redshift of $z \simeq 0.736$ (Xu et al. 2016). In particular, the X-ray afterglow monitored by Swift-XRT exhibited a prominent shallow decay phase (D'Avanzo et al. 2016), which adds credibility to a magnetar central engine. In addition, the burst duration during which the observed (gamma-ray and X-ray) flux is dominated by jet emission is $t_{\text {burst }} \simeq 10^{3} \mathrm{~s}$ (Zhang et al. 2014; Gao et al. 2017), which is also in favor of a longlasting magnetar inside. Therefore, we consider GRB $160804 \mathrm{~A}$ as a perfect candidate and we will discuss in detail here.

\subsection{Prompt Emission of GRB 160804A}

GRB 160804A triggered Fermi-GBM at $T_{0}=01: 34$ : 16.04 UT on 04 August 2016 and the public data is processed using a pipeline described in Zhang et al. (2016). Since the prompt phase lasts over 100 seconds, we need

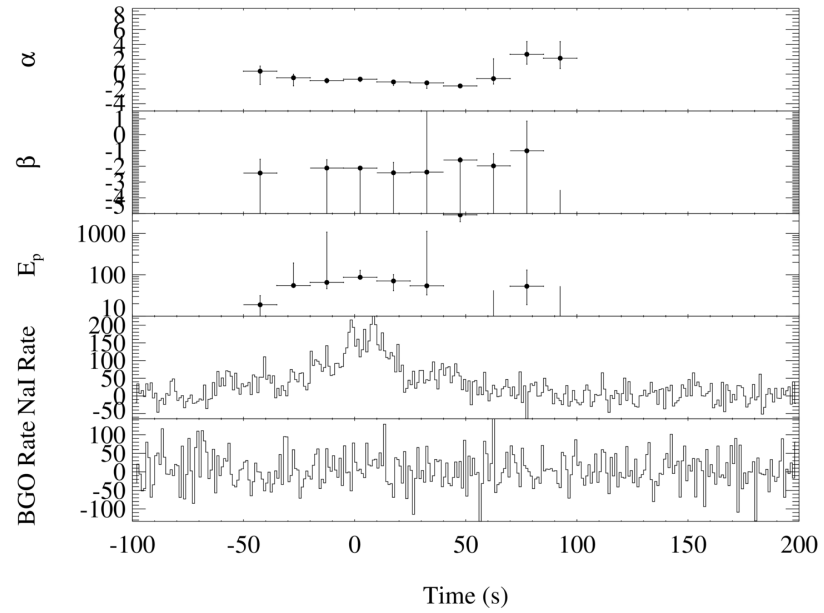

FIG. 1.- The time-resolved spectrum fitting results with the Band function. The top three panels show the evolution of three parameters $\alpha, \beta, E_{p}$ defined in the Band function during ten time intervals. The bottom two panels show the light curves of $\mathrm{NaI}$ and BGO detectors on board Fermi.

to do a time-resolved spectral analysis. As a trial, we binned the light curve into ten time intervals equally, starting from $T_{0}-50 \mathrm{~s}$ to $T_{0}+100 \mathrm{~s}$. In each time interval, the spectrum is preliminarily fitted by the Band function and the evolutions of its parameters $\left(\alpha, \beta, E_{p}\right)$ are shown in Figure 1. We can see that $\alpha$ is close to -1 and $E_{p}$ is several tens of $\mathrm{keV}$ in most time intervals. We note that the last four intervals are dominated by background photons so the Band function parameters are unconstrained. We thus only focus on the first six time intervals in our analysis.

In order to test the MIGMAD model, we need to interpret the prompt emission properties of GRB 160804A. We fit the first six time intervals using the MIGMAD model with McSpecFit package (Zhang et al. 2016). Spectral fitting is then performed within the allowed range via a Bayesian Monte-Carlo method. In a typical manner, Figure 2 shows the parameter corner plot of interval-3 (main peak of the light curve). The spectrum fitting results are shown in Figure 3 and 4, while the bestfitting parameters are listed in Table 1 . As we can see clearly, the MIGMAD model fits the observed spectrum well, with PGSTAT/d.o.f close to unity. Furthermore, thermal components that are predicted in the MIGMAD model are clearly seen in all six intervals. Moreover, we can plot the time evolutions of these five parameters in Figure 5, which give a lot of information about the central magnetar that will be discussed later in section 3.3.

\subsection{X-ray Afterglow}

The X-ray afterglow data of GRB 160804A was recorded $137 \mathrm{~s}$ after the BAT trigger, and it shows a prominent shallow decay phase without obvious Xray flares (D'Avanzo et al. 2016). The shallow decay of X-ray afterglow is commonly ascribed to the energy injection from a central magnetar (Dai \& Lu 1998a,b; Zhang \& Mészáros 2001). We follow the standard procedure described in Yu et al. (2009, 2010), in which the $\mathrm{X}$-ray light curve after the initial steep decay phase was 


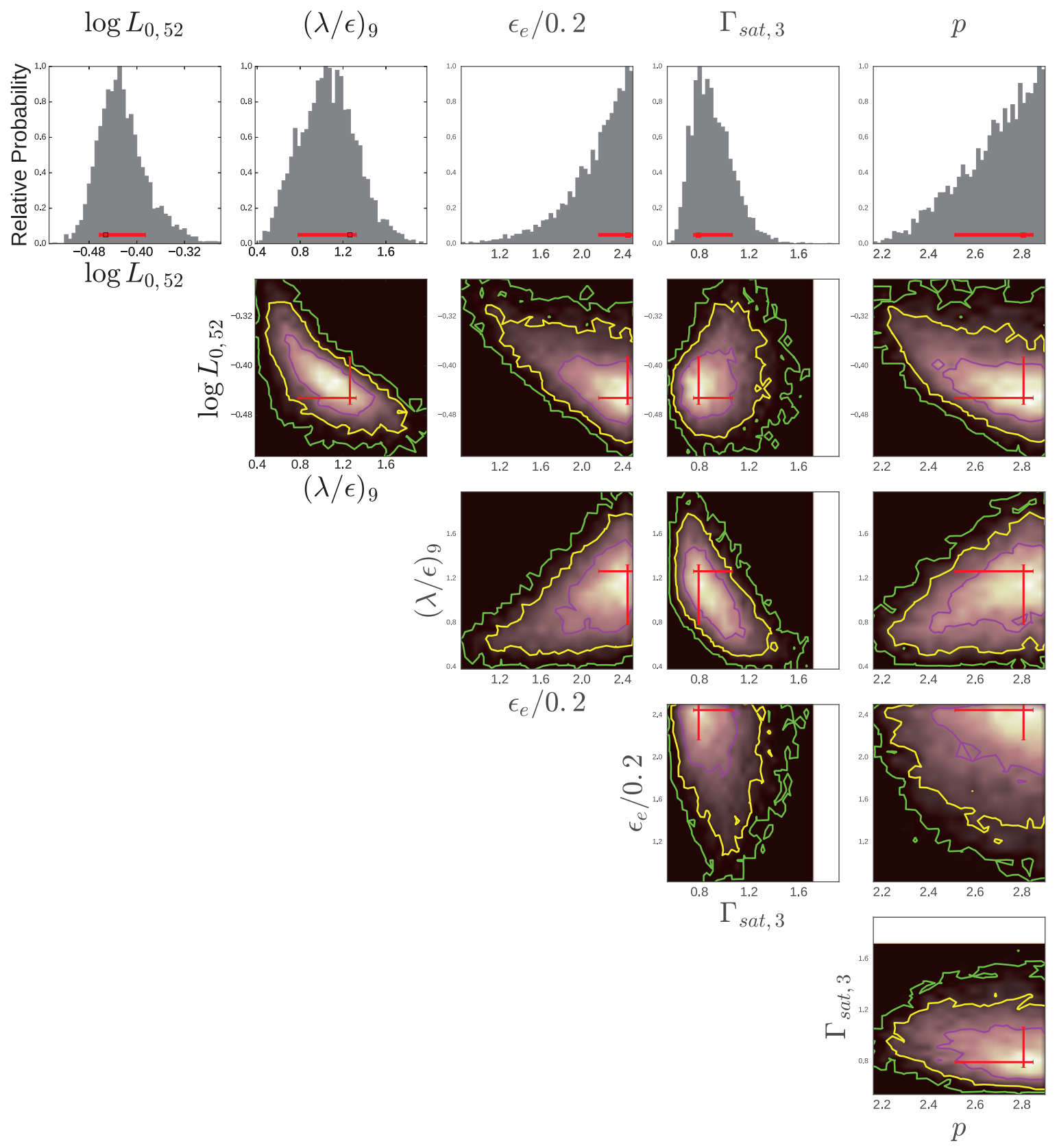

FIG. 2.- Parameter constraints of the MIGMAD model fitting for the spectrum in time interval-3 (from $-5 \mathrm{~s}$ to $10 \mathrm{~s}$ ). Histograms and contours illustrate the likelihood map. Red crosses show the best-fitting values and 1-sigma error bars.

fitted by a smoothed double broken power-law function,

$$
\begin{aligned}
F_{X}(t)= & F_{X, b}\left[\left(\frac{t}{T_{b 1}}\right)^{\omega \alpha_{1}}+\left(\frac{t}{T_{b 1}}\right)^{\omega \alpha_{2}}\right. \\
& \left.+\left(\frac{T_{b 2}}{T_{b 1}}\right)^{\omega \alpha_{2}}\left(\frac{t}{T_{b 2}}\right)^{\omega \alpha_{3}}\right]^{-1 / \omega},
\end{aligned}
$$

where typical $w=3$ is assumed (Liang et al. 2007). The fitting is performed using McEasyFit (Zhang et al. 2016) and the result is shown in Figure 6, with the best-fitting values being listed in Table 2 . In view of the late-time upper limit from Swift-XRT, a double power-law function generally fits better than a single broken power-law one. This fitting could also have some implications for the magnetar and will be discussed in the following section.

\subsection{Implications for the Central Magnetar}


TABLE 1

Best-Fitting Parameters With the MiGMAD MODEL FOR SiX time-INTERVALS of GRB160804A

\begin{tabular}{|c|c|c|c|c|c|c|c|}
\hline \multirow[b]{2}{*}{ Parameter } & \multirow[b]{2}{*}{ Allowed range } & \multicolumn{6}{|c|}{ Best-fitting value } \\
\hline & & $\begin{array}{c}\text { interval-0 } \\
{[-50 \mathrm{~s},-35 \mathrm{~s}]}\end{array}$ & $\begin{array}{c}\text { interval-1 } \\
{[-35 \mathrm{~s},-20 \mathrm{~s}]}\end{array}$ & $\begin{array}{l}\text { interval-2 } \\
{[-20 \mathrm{~s},-5 \mathrm{~s}]}\end{array}$ & $\begin{array}{l}\text { interval-3 } \\
{[-5 \mathrm{~s}, 10 \mathrm{~s}]}\end{array}$ & $\begin{array}{l}\text { interval-4 } \\
{[10 \mathrm{~s}, 25 \mathrm{~s}]}\end{array}$ & $\begin{array}{l}\text { interval-5 } \\
{[25 \mathrm{~s}, 40 \mathrm{~s}]}\end{array}$ \\
\hline $\log L_{0,52}$ & {$[-5,0]$} & $-1.19_{-0.19}^{+0.12}$ & $-0.61_{-0.18}^{+0.08}$ & $-0.82_{-0.01}^{+0.13}$ & $-0.45_{-0.01}^{+0.07}$ & $-0.63_{-0.12}^{+0.13}$ & $-0.81_{-0.10}^{+0.21}$ \\
\hline$(\lambda / \epsilon)_{9}$ & {$[0.1,3.0]$} & $2.91_{-0.64}^{+0.09}$ & $2.71_{-0.79}^{+0.29}$ & $2.50_{-0.64}^{+0.50}$ & $1.26_{-0.48}^{+0.06}$ & $1.24_{-0.18}^{+0.95}$ & $2.15_{-0.64}^{+0.54}$ \\
\hline$\epsilon_{e} / 0.2$ & {$[0.25,2.5]$} & $0.94_{-0.09}^{+1.22}$ & $0.70_{-0.20}^{+0.68}$ & $2.44_{-0.42}^{+0.06}$ & $2.44_{-0.28}^{+0.05}$ & $1.46_{-0.28}^{+0.84}$ & $1.18_{-0.27}^{+1.01}$ \\
\hline$\Gamma_{\text {sat }, 3}$ & {$[0.1,2.0]$} & $0.32_{-0.08}^{+1.22}$ & $1.49_{-0.92}^{+0.12}$ & $0.51_{-0.21}^{+0.26}$ & $0.79_{-0.04}^{+0.27}$ & $1.04_{-0.21}^{+0.36}$ & $0.82_{-0.40}^{+0.40}$ \\
\hline$p$ & {$[2.1,2.9]$} & $2.32_{-0.08}^{+0.46}$ & $2.11_{-0.01}^{+0.28}$ & $2.83_{-0.21}^{+0.07}$ & $2.81_{-0.29}^{+0.04}$ & $2.61_{-0.36}^{+0.18}$ & $2.87_{-0.26}^{+0.03}$ \\
\hline \multicolumn{2}{|c|}{ PGSTAT/d.o.f } & $299.2 / 361.0$ & $311.6 / 361.0$ & $312.5 / 361.0$ & $337.2 / 361.0$ & $298.1 / 361.0$ & $303.3 / 361.0$ \\
\hline
\end{tabular}
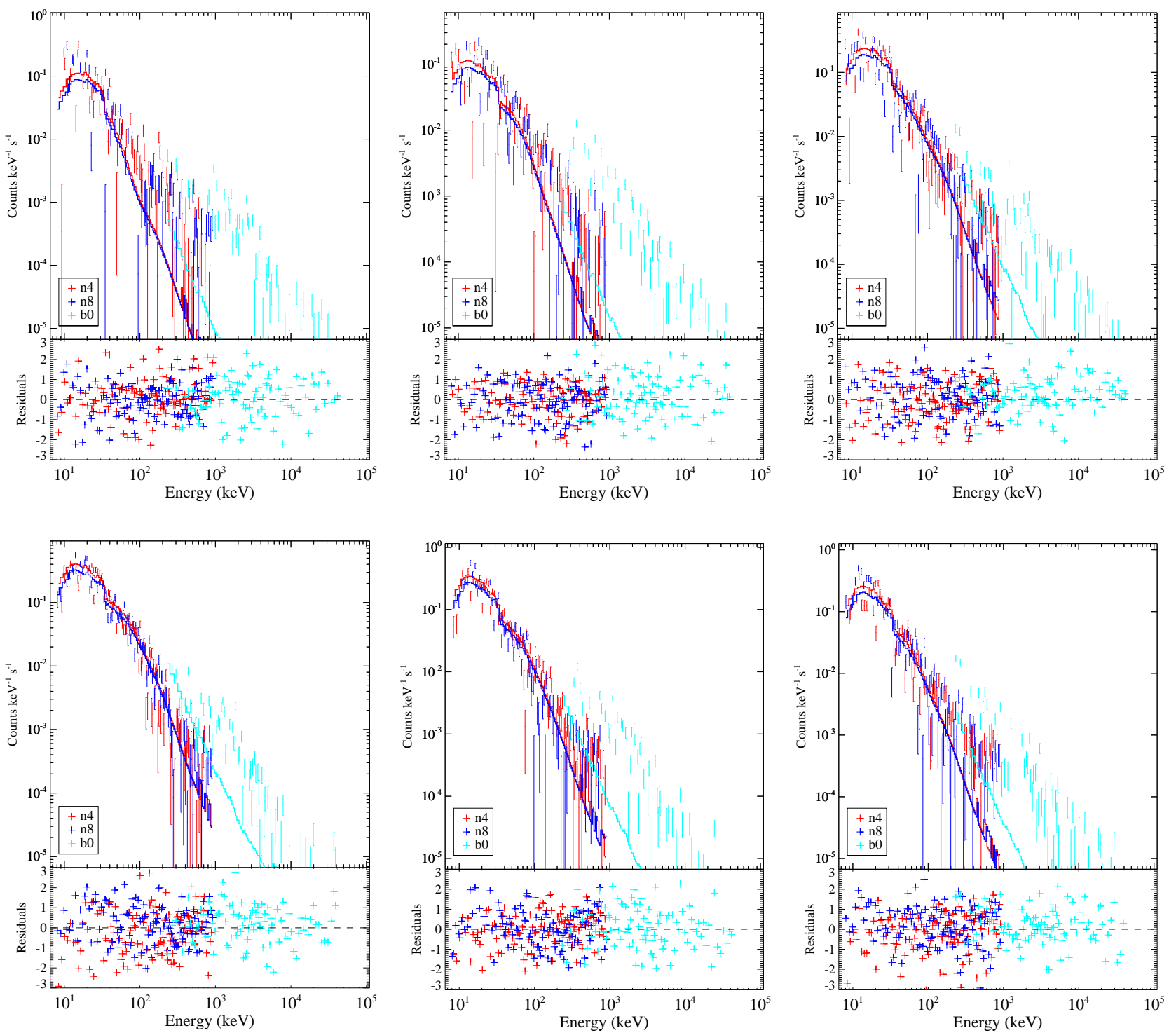

FIG. 3.- The observed count spectrum and model count spectrum for six time intervals. n4, n8, b0 represent three Fermi/GBM detectors. From left to right: first row - intervals $0,1,2$; second row $-3,4,5$. 

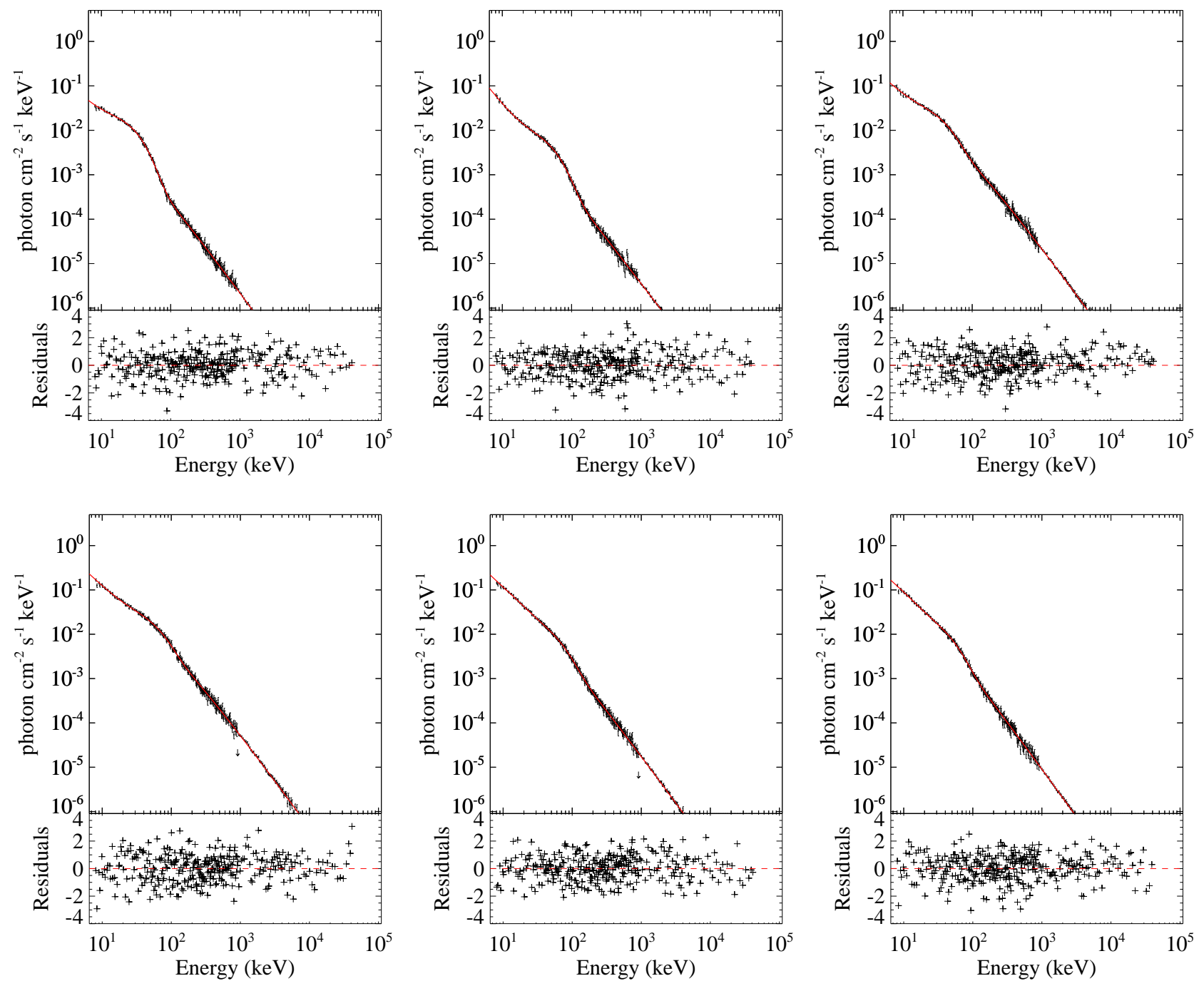

Fig. 4.- Same as Figure 3 but for the observed photon flux (black datapoints) and theoretical photon spectrum (red line). 


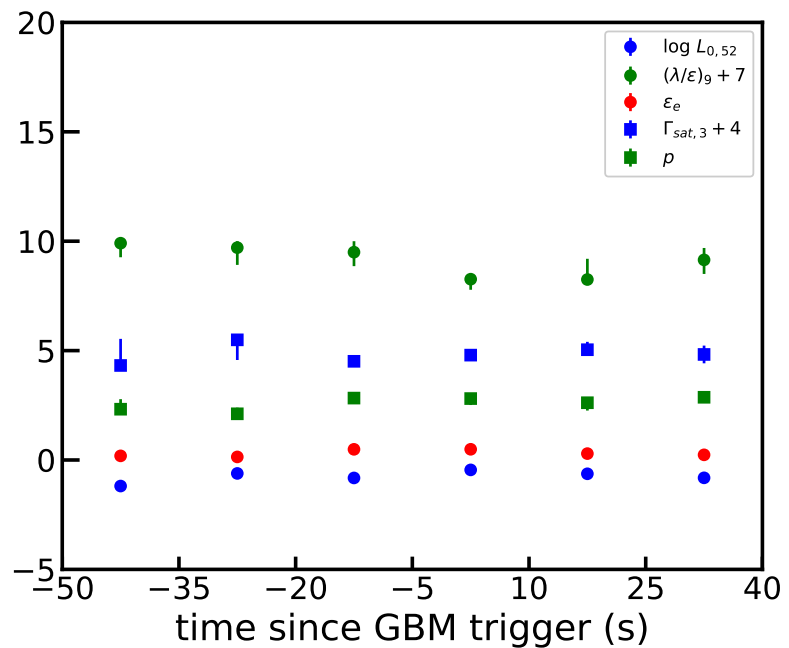

FIG. 5.- The time evolution of best-fitting parameters with the MIGMAD model for GRB 160804A. To avoid possible overlapping, the value of $(\lambda / \epsilon)_{9}$ is added with 7 and $\Gamma_{\text {sat,3 }}$ is added with 4 in the figure.

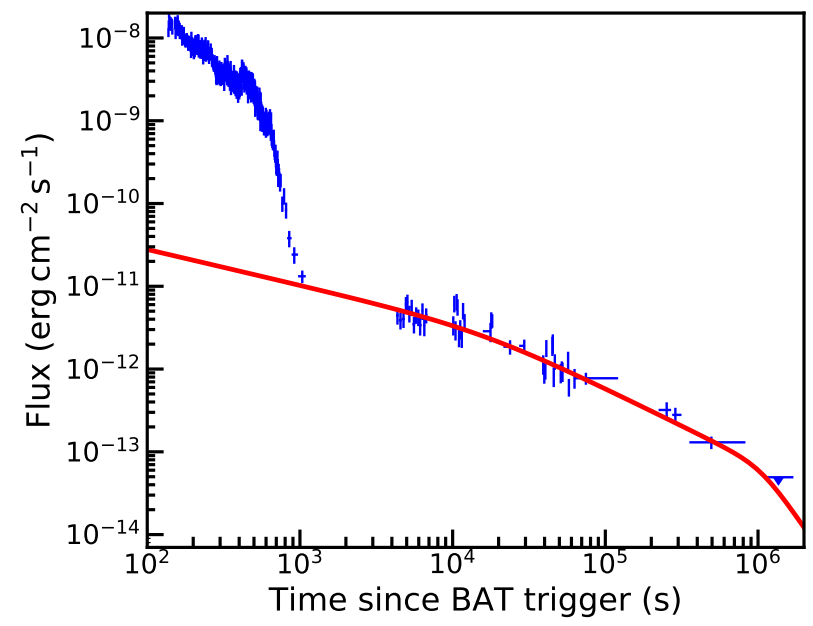

FIG. 6.- Fitting the X-ray afterglow light curve of GRB 160804A (blue datapoints) with double broken power law (red line) function. A second break is needed to reconcile the last upper limit from observation.

Firstly, for a double power-law type afterglow, the two break times are believed to be correlated to the magnetar spin evolution (Yu et al. 2010). Before the first break, the gravitational wave radiation dominates the spin-down and its typical timescale is $T_{g} \sim T_{b 1} /(1+z)$. For GRB $160804 \mathrm{~A}$, we get $T_{g} \simeq 9.35_{-3.62}^{+19.05} \times 10^{3} \mathrm{~s}$. The other break time is attributed to a break of the magnetic dipole luminosity at $T_{c} \sim T_{b 2} /(1+z) \simeq 2.53_{-0.40}^{+2.49} \times 10^{5} \mathrm{~s}$ and the typical magnetic braking timescale is $T_{m}=$ $\left(T_{g} T_{c}\right)^{1 / 2} \simeq 4.87_{-1.37}^{+7.08} \times 10^{4} \mathrm{~s}$. Since the initial period
TABLE 2

The BeST-FITTING VALUES FOR THE SIX PARAMETERS DEFINED IN EQUATION (12).

\begin{tabular}{ccc}
\hline Parameter & Allowed range & Best-fitting value \\
\hline$F_{X, b}$ & {$\left[10^{-15}, 10^{-10}\right]$} & $3.09_{-1.69}^{+1.14} \times 10^{-12}$ \\
$\alpha_{1}$ & {$[-1.0,1.0]$} & $0.91_{-0.48}^{+0.02}$ \\
$\alpha_{2}$ & {$[0.0,2.0]$} & $0.43_{-0.03}^{+0.51}$ \\
$\alpha_{3}$ & {$[2.0,3.0]$} & $2.71_{-0.55}^{+0.11}$ \\
$T_{b 1}$ & {$\left[10^{3}, 10^{5}\right]$} & $1.62_{-0.63}^{+3.03} \times 10^{4}$ \\
$T_{b 2}$ & {$\left[10^{4}, 10^{6}\right]$} & $4.40_{-0.70}^{+4.32} \times 10^{5}$ \\
\hline
\end{tabular}

and magnetic field is given by

$$
\begin{array}{r}
P_{i,-3}=a^{-1 / 6} T_{g, 3}^{1 / 6}, \\
B_{i, 14}=1.4 P_{i,-3} T_{m, 5}^{-1 / 2},
\end{array}
$$

where $a$ is a slow-varying numerical factor of order unity (Yu et al. 2009, 2010), we can deduce that $P_{i}=$ $1.45_{-0.11}^{+2.30} \mathrm{~ms}$ and $B_{i}=2.91_{-0.68}^{+0.26} \times 10^{14} \mathrm{G}$ for the magnetar of GRB 160804A.

Secondly, we can get some information of the central magnetar from the prompt emission. In the prompt phase $\left(T_{90} \ll T_{g}\right)$, gravitational braking dominates the spin-down. Since the energy loss rate of gravitational radiation is in proportion to the six power of angular velocity (Shapiro \& Teukolsky 1983), the evolution of angular velocity can be obtained by solving the spin evolution equation, which gives

$$
\Omega(t)=\Omega_{i}\left(1+\frac{4 \Omega_{i}^{4}}{\Omega_{s}^{4}} \frac{\left(t+t_{0}\right)}{T_{g}}\right)^{-1 / 4},
$$

where $\Omega_{i}, \Omega_{s}$ represent the initial, the stable angular velocity after gravitational braking respectively. We introduce a parameter $t_{0}$ here to account for the possible time delay between the birth of magnetar and the GRB trigger. This is naturally expected since the magnetar should cool first and jet break-out also takes some time (Metzger et al. 2011). The spin period is then $P(t)=2 \pi / \Omega(t)$ and the evolution of parameter $(\lambda / \epsilon)$ can be obtained as constant $\epsilon=0.1$ is assumed. Moreover, the magnetic dipole luminosity is $L_{m}(t)=B^{2} R^{6} \Omega^{4} / 6 c^{3}$. If the magnetic field and jet beaming factor $f_{B}=(1-$ $\left.\cos \theta_{j}\right) \simeq \theta_{j}^{2} / 2$ do not vary with time, theoretically we have $L_{0}(t)=(1 / 4 \pi) L_{m} / f_{B}=(1 / 4 \pi) B^{2} R^{6} /\left(6 c^{3} f_{B}\right) \times$ $\Omega(t)^{4} \equiv K \Omega(t)^{4}$. Taking $\Omega_{i}, \Omega_{s}, t_{0}, K$ as parameters, we can perform a combined fit of time evolution for parameters $L_{0}$ and $(\lambda / \epsilon)$ that obtained in Section 3.1. The fitting results are shown in Figure 7 and Table 3. As we can see, the initial spin period deduced from prompt emission fitting is $P_{i}=2 \pi / \Omega_{i}=1.43_{-0.30}^{+0.27} \mathrm{~ms}$, which matches perfectly with the value of $P_{i}=1.45_{-0.11}^{+2.30} \mathrm{~ms}$ from afterglow fitting in section 3.2 . These two independent approaches giving a consistent result adds great credibility to the MIGMAD model and a millisecond magnetar central engine. Note that the theoretical curves in 
TABLE 3

THE BEST-FITTING VALUES FOR THE FOUR PARAMETERS DEFINED IN EQUATION (14).

\begin{tabular}{ccc}
\hline Parameter & Allowed range & Best-fitting value \\
\hline$\Omega_{i}$ & {$\left[3 \times 10^{3}, 6 \times 10^{3}\right]$} & $4.40_{-0.69}^{+1.18} \times 10^{3}$ \\
$\Omega_{0}$ & {$[6,600]$} & $492.16_{-7.78}^{+14.22}$ \\
$t_{0}$ & {$[50,150]$} & $50.04_{-0.03}^{+1.54}$ \\
$K$ & {$\left[10^{35}, 6 \times 10^{38}\right]$} & $5.98_{-0.13}^{+0.01} \times 10^{38}$ \\
\hline
\end{tabular}

Figure 7 are obtained just through the time evolution of spin period, while Beniamini et al. (2017) considered time evolutions of several other quantities (e.g, dipole field strength, baryon loading, etc).

Lastly, more details can be deduced if we further do a comprehensive analysis. On one hand, equation (13) gives a best-fitting magnetic field $B_{i}=2.91 \times 10^{14} \mathrm{G}$. On the other hand, from equation (1) we can get that magnetic field $B \propto r^{-4 / 3}$ in the Poynting-flux dominated jet. Taking time interval-3 (main peak) as a representative, tracing back to a position near the surface of the magnetar, we can get $B(r)=6.13 \times 10^{14} r_{6}^{-4 / 3} \mathrm{G}^{2}$. Comparing with the field strength from afterglow fitting, the radius of the magnetar can be determined as $R=1.75 \times 10^{6} \mathrm{~cm}$. Since $K \equiv B^{2} R^{6} /\left(6 c^{3} f_{B}\right)$, substituting the best-fitting value of $K$ and $R$ we can get $f_{B}=2 \times 10^{-6}$, which corresponds to a narrowly beamed jet with half opening angle $\theta_{j} \simeq 0.002$. This value is much smaller than the opening angles that are usually deduced from afterglow jet-break signatures. On one hand, for these extremely narrow jets, the jet-break may happen at very early times (e.g. Frail et al. 2001), which are not easy to be identified since the X-ray afterglows may be still in a steep decay phase. This may explain why so many afterglows do not show jet-break signatures. In this sense, observationally there may exist a bias, since jet-break phenomena are easier to be identified for jets with larger opening angles. On the other hand, for a magnetized jet, during propagating in the progenitor envelope, the jet head will contract due to strong hoop stress, which gives rise to a nozzle-like jet shape after breakout (Bromberg et al. 2014). Basically, the opening angle of the magnetized jet could be much smaller than that of the hydrodynamic jet. In the recent numerical simulation work (Bromberg \& Tchekhovskoy 2016) we can see that the magnetized jet could be very slim (Figure 15 in their paper). Also, the opening angle depends on the density profile of medium. A denser envelope generally leads to a smaller jet opening angle. In view of these arguments above, the peculiar $\theta_{j}=0.002$ for this GRB 160804A is still reasonable, though not very

\footnotetext{
2 Strictly speaking, the Poynting-flux dominated outflow originate near the light cylinder (Aharonian et al. 2012), at a radius $R_{L}=c / \Omega \simeq 4.78 \times 10^{6} \mathrm{~cm}$ for a typical millisecond magnetar. However, the dipole magnetic field decays with a radius follows $B \propto r^{-b}$ where $\mathrm{b}$ is between 1 (toroidal) and 2 (poloidal). Thus, tracing back to light cylinder or the magnetar surface will not make a significant difference in the calculation.
}

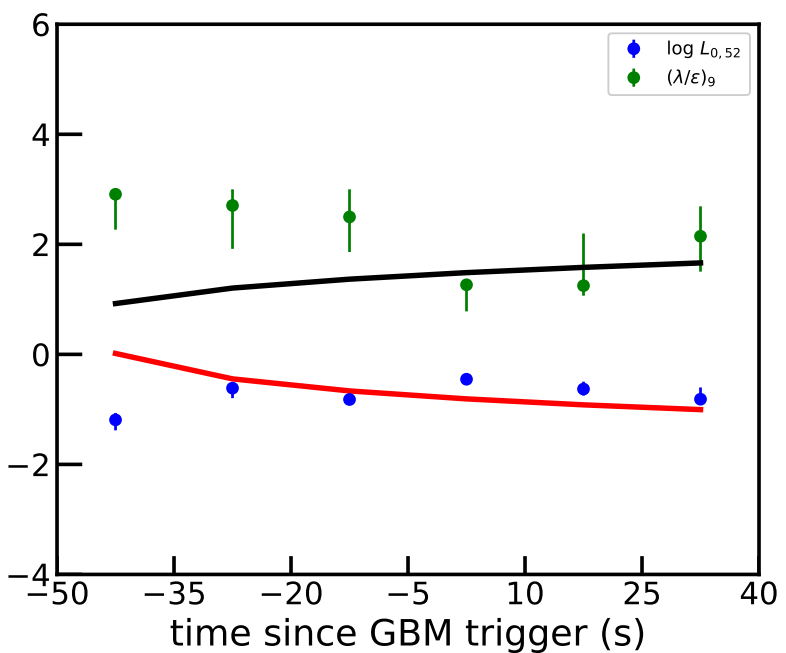

FIG. 7. - The theoretical time evolutions (red line and black line) for the two spectral fitting parameters $L_{0,52}$ (blue points) and $(\lambda / \epsilon)_{9}$ (green points) in the MIGMAD model.

typical.

\section{DISCUSSIONS AND CONCLUSIONS}

In this work we have revisited the scenario for the prompt emission of GRB, called the MIGMAD model, by assuming that the central engine is a newborn millisecond magnetar, whose wind undergoes an internal gradual magnetic dissipation. This model is relatively clean and has only few assumptions. Theoretically, we have investigated the high-energy emission from the wind of this newborn magnetar, which should exist as long as the wind is initially Poynting-flux dominated. We have applied it to the prompt emission of GRB 160804A and showed that its spectrum can be fitted very well. We found that a beamed wind with half opening angle 0.01 from a newborn magnetar that has an initial spin period $1.45 \mathrm{~ms}$ and surface magnetic field $2.91 \times 10^{14} \mathrm{G}$ can reproduce the prompt emission and afterglow behaviors well. In addition, we can also predict a subclass of GRBs that are produced based on this scenario, which are named as "MIGMAD bursts".

MIGMAD bursts should have distinctive properties compared to normal ones. First, a general testable prediction of the MIGMAD model is that the peak of the flux spectrum determined by the photospheric component is usually around a hundred $\mathrm{keV}$ and not so sensitive to the model parameters (Beniamini \& Giannios 2017). Second, the most prominent property is that the durations of MIGMAD bursts might be very long (up to $\sim 10^{2}-10^{4} \mathrm{~s}$ ), depending mainly on the spindown timescale of the central magnetar. Actually, we know little about the production of ultra-long GRBs (ULGRBs) now. Existing models for ULGRBs include collapses of blue supergiant stars (Gendre et al. 2013; Levan et al. 2014), newborn magnetars (Greiner et al. 2015; Metzger et al. 2018), white dwarf tidal disruption events (Ioka et al. 2016) and black hole hyper-accretion processes (Liu et al. 2018). Especially, Greiner et al. (2015) claimed that the associated supernova (SN $2011 \mathrm{kl}$ ) of ULGRB 111209A is powered by a magnetar. 
However, the radiation mechanism of ULGRB has not been detailedly discussed in their work. In this work we show that MIGMAD scenario is able to explain the prompt emission properties, making the magnetar origin model concrete and complete. In this sense, we might already have some other interesting candidates like GRB 111209A and GRB 060218, which will be investigated in our future work. Once the deduced spin period and magnetic field can match well from the independent analysis of prompt emission, afterglow and associated supernova observations for one GRB, its magnetic central engine would be finally fully convinced.

Due to the limited available spin-down luminosity of a millisecond magnetar and the low efficiency in converting magnetic energy to radiation by gradual dissipation (Xiao \& Dai 2017), given the current Fermi-GBM sensitivity we can only detect relatively nearby MIGMAD bursts with redshift less than 1. Even though, FermiGBM should have caught a few events so that it is mean- ingful to look for candidates in the archival data. More facilities with better sensitivities (e.g. Insight-HXMT) would be able to detect other more MIGMAD bursts in the near future.

This work was supported by the National Basic Research Program of China (973 Program, Grant No. 2014CB845800), the National Key Research and Development Program of China (Grant No. 2017YFA0402600) and the National Natural Science Foundation of China (Grant No. 11573014). DX was also supported by the Natural Science Foundation for the Youth of Jiangsu Province (Grant NO. BK20180324) and the Fundamental Research Funds for the Central Universities (Grant No. 020114380027). BBZ acknowledge the support from the National Thousand Young Talents program of China. We acknowledge the use of the public data from the Fermi satellite.

\section{REFERENCES}

Aharonian, F. A., Bogovalov, S. V., \& Khangulyan, D. et al. 2012, Nature, 482, 257.

Atoyan, A. M. 1999, A\&A, 346, L49

Bégué, D. \& Pe'er, A. 2015, ApJ, 802, 134

Beloborodov, A. M. 2010, MNRAS, 407, 1033

Beniamini, P., \& Piran, T. 2013, ApJ, 769, 69

Beniamini, P., \& Piran, T. 2014, MNRAS, 445, 3892

Beniamini, P., Giannios, D., \& Metzger, B. D. 2017, MNRAS, 472, 3058

Beniamini, P., \& Giannios, D. 2017, MNRAS, 468, 3202

Beniamini, P., \& Mochkovitch, R. 2017, A\&A, 605, 60

Beniamini, P., Barniol Duran, R., \& Giannios, D. 2018, MNRAS, 476, 1785

Bissaldi, E., Hui, C. M., Connaughton, V., et al. 2016, GCN Circular 19769

Bošnjak, Ž., Daigne, F., \& Dubus, G. 2009, A\&A, 498, 677

Bromberg, O., Nakar, E., Piran, T., \& Sari, R. 2011, ApJ, 740 100

Bromberg, O., Granot, J., Lyubarsky, Y., \& Piran, T. 2014, MNRAS, 443, 1532

Bromberg, O., Tchekhovskoy, A. 2016, MNRAS, 456, 1739

Coroniti, F. V. 1990, ApJ, 349, 538

Dai, Z. G., \& Lu, T. 1998, A\&A, 333, L87

Dai, Z. G., \& Lu, T. 1998, Phys. Rev. Lett., 81, 4301

Dai, Z. G., Daigne, F., \& Mészáros, P. 2017, Space Sci. Rev., 212, 409

Daigne, F., \& Mochkovitch, R. 1998, MNRAS, 296, 275

Daigne, F., Bošnjak, Z., \& Dubus, G. 2011, A\&A, 526, A110

D'Avanzo, P., D'Elia, V., Roegiers, T. G. R., et al. 2016, GCN Circular 19763

Deng, W., \& Zhang, B. 2014, ApJ, 785, 112

Derishev 2007, Astrophysics and Space Science, 308, 157

Drenkhahn, G. 2002, A\&A, 387, 714

Drenkhahn, G., \& Spruit, H. C. 2002, A\&A, 391, 1141

Faber, J. A., \& Rasio, F. A. 2012, LRR, 15, 8

Frail, D. A., Kulkarni, S., Sari, R., et al. 2001, ApJL, 562, L55

Gao, H., \& Zhang, B. 2015, ApJ, 801, 103

Gao, H., Ren, A.-B., Lei, W.-H., et al. 2017, ApJ, 845, 51

Geng, J. J., Huang, Y. F., Wu, X. F., et al. 2018a, ApJS, 234, 3

Gendre, B., Stratta, G., Atteia, J. L., et al. 2013, ApJ, 766, 30

Ghisellini, G., \& Celotti, A. 1999, ApJ, 511, 93

Giannios, D., \& Spruit, H. C. 2005, A\&A, 430, 1

Giannios, D. 2010, MNRAS, 408, L46

Giannios, D. 2012, MNRAS, 422, 3092

Greiner J., Mazzali, P. A., Kann, D. A., et al. 2015, Nature, 523, 189

Guo, F., Liu, Y. H., Daughton, W., \& Li, H. 2015, ApJ, 806, 167

Huang, Y. F., Dai, Z. G., \& Lu, T. 1999, MNRAS, 309, 513

Ioka, K., Hotokezaka, K., \& Piran, T. 2016, ApJ, 833, 110

Kagan, D., Sironi, L., Cerutti, B., \& Giannios, D. 2015,

Space Sci. Rev., 191, 545

Kobayashi, S., Piran, T., \& Sari, R. 1997, ApJ, 490, 92
Kumar, P. \& McMahon, E. 2008, MNRAS, 384, 33

Kumar, P., \& Zhang, B. 2015, Physics. Reports, 561, 1

Lei, W. H., Zhang, B., \& Liang, E. W. 2013, ApJ, 765, 125

Lemoine, M. 2013, MNRAS, 428, 845

Levan, A. J., Tanvir, N. R., Starling, R. L. C., et al. 2014, ApJ, 781,13

Liang, E. W., Zhang, B. B., \& Zhang, B. 2007, ApJ, 670, 565

Liu, Y. H., Guo, F., Daughton, W., Li, H., \& Hesse, M. 2015, Phys. Rev. Lett., 114, 5002

Liu, T., Gu, W. M., \& Zhang, B. 2017, NewAR, 79, 1

Liu, T., Song, C. Y., Zhang, B., et al. 2018, ApJ, 852, 20

Lundman, C., Pe'er, A., \& Ryde, F. 2013, MNRAS, 428, 2430

Lü, H. J., Zou, L., Lan, L., Liang, E. W. 2018, MNRAS, 480, 4402

Marshall, F. E., D'Avanzo, P., Kennea, J. A., et al. 2016, GCN Circylar 19761

Metzger, B. D., Giannios, D., Thompson, T. A., Bucciantini, N., \& Quataert, E. 2011, MNRAS, 413, 2031

Metzger, B. D., Beniamini, P., Giannios, D. 2018, ApJ, 857, 95

Pe'er, A., Mészáros, P., \& Rees, M. J. 2005, ApJ, 635, 476

Rees, M. J., \& Mészáros, P. 1994, ApJL, 430, L93

Rees, M. J., \& Mészáros, P. 2005, ApJ, 628, 847

Rosswog, S., Ramirez-Ruiz, E., \& Davies, M. B. 2003, MNRAS, 345, 1077

Ruffert, M., Janka, H. T., Takahashi, K., \& Schäfer, G. 1997, A\&A, 319, 122

Sari, R., Piran, T., Narayan, R. 1998, ApJL, 497, L17

Shapiro, S. L., \& Teukolsky, S. A. 1983, JBAA, 93, 276

Sironi, L., \& Spitkovsky, A. 2014, ApJ, 783, L21

Spruit, H. C., Daigne, F., \& Drenkhahn, G. 2001, A\&A, 369, 694

Thompson, C. 1994, MNRAS, 270, 480

Uhm, Z. L., \& Zhang, B. 2014, Nature Physics, 10, 351

Usov, V. V. 1992, Nature, 357, 472

Werner, G. R., Uzdensky, D. A., Cerutti, B., Nalewajko, K., \& Begelman, M. C. 2016, ApJL, 816, L8

Wu, X. F., Hou, S. J., \& Lei, W. H. 2013, ApJL, 767, L36

Xiao, D. \& Dai, Z. G. 2017, ApJ, 846, 130

Xu, D., Heintz, K. E., Malesani, D., et al. 2016, GCN Circular 19773

Yu, Y. W., Cao, X. F., \& Zheng, X. P. 2009, ApJ, 706, 221

Yu, Y. W., Cheng, K. S., \& Cao, X. F. 2010, ApJ, 715, 477

Zhang, B., Fan, Y. Z., Dyks, J., et al. 2006, ApJ, 642, 354

Zhang, B., \& Mészáros, P. 2001, ApJ, 552, L35

Zhang, B., \& Yan, H. 2011, ApJ, 726, 90

Zhang, B. -B., Zhang, B., Murase, K., et al. 2014, ApJ, 787, 66

Zhang, B. -B., Uhm, Z. L., Connaughton, V., et al. 2016, ApJ, 816,72

Zhang, D., \& Dai, Z. G. 2008, ApJ, 683, 329

Zhang, D., \& Dai, Z. G. 2009, ApJ, 703, 461

Zhang, D., \& Dai, Z. G. 2010, ApJ, 718, 841 